\title{
REMARKS ON NEARLY EQUIVALENT OPERATORS
}

\author{
EungIL Ko AND MEE-Jung LeE
}

Abstract. An operator $S \in \mathscr{L}(\mathscr{H})$ is said to be nearly equivalent to $T$ if there exists an invertible operator $V \in \mathscr{L}(\mathscr{H})$ such that $S^{*} S=V^{-1} T^{*} T V$. In this paper, we study several properties of nearly equivalent operators, and investigate their local spectral properties and invariant subspaces.

Mathematics subject classification (2010): 47B20, 47A10.

Keywords and phrases: Nearly equivalent operators, local spectral property, invariant subspace.

\section{REFERENCES}

[1] P. AiEnA, Fredholm and local spectral theory with applications to multipliers, Kluwer Acad. Pub., 2004.

[2] E. Albrecht and J. Eschmeier, Analytic functional models and local spectral theory, Proc. London Math. Soc. 75 (1997), 323-348.

[3] C. BERGER, Sufficiently high powers of hyponormal operators have rationally invariant subspaces, Inter. Equ. Oper. Th. 1 (1978), 444-447.

[4] S. BRown, Hyponormal operators with thick spectrum have invariant subspaces, Ann. of Math. 125 (1987), 93-103.

[5] I. ColojoARA AND C. FoiAs, Theory of generalized spectral operators, Gordon and Breach, New York, 1968.

[6] I. B. Jung, E. Ko, And C. PeArcy, Aluthge transforms of operators, Inter. Equ. Oper. Th. 37 (2000), 449-456.

[7] E. Ko And M. KIm, Some connections between an operator and its Aluthge transform, Glasgow Math. J. 47 (2005), 167-175.

[8] K. Laursen and M. Neumann, An introduction to local spectral theory, Clarendon Press, Oxford, 2000.

[9] S. I. Othman, Nearly equivalent operators, Math. Bohem. 121 (1996), 133-141.

[10] H. RADJAVi AND P. Rosenthal, Invariant subspaces, Springer-Verlag, 1973.

[11] D. XIA, Spectral theory of hyponormal operators, Op. Th.: Adv. Appl. 10, Birkhäuser Verlag, Boston, 1983. 